\title{
Characterization of aerosol particles at Cape Verde close to sea and cloud level heights - Part 2: ice nucleating particles in air, cloud and seawater
}

Xianda Gong ${ }^{1}$, Heike Wex ${ }^{1}$, Manuela van Pinxteren ${ }^{1}$, Nadja Triesch ${ }^{1}$, Khanneh Wadinga Fomba ${ }^{1}$, Jasmin Lubitz ${ }^{1}$, Christian Stolle ${ }^{2,3}$, Tiera-Brandy Robinson ${ }^{3}$, Thomas Müller ${ }^{1}$, Hartmut Herrmann ${ }^{1}$, and Frank Stratmann ${ }^{1}$

${ }^{1}$ Leibniz Institute for Tropospheric Research, Leipzig, Germany

${ }^{2}$ Leibniz-Institute for Baltic Sea Research Warnemünde (IOW), Rostock, Germany

${ }^{3}$ Institute for Chemistry and Biology of the Marine Environment, University of Oldenburg, Wilhelmshaven, Germany

Correspondence: Xianda Gong (gong@tropos.de) 


\section{S1 Seawater samples}

Table S1. The information of seawater samples at OS, including sample number, start time, end time, location, salinity, sodium chloride $(\mathrm{NaCl})$ mass concentration, $\mathrm{PH}$ value and water temperature.

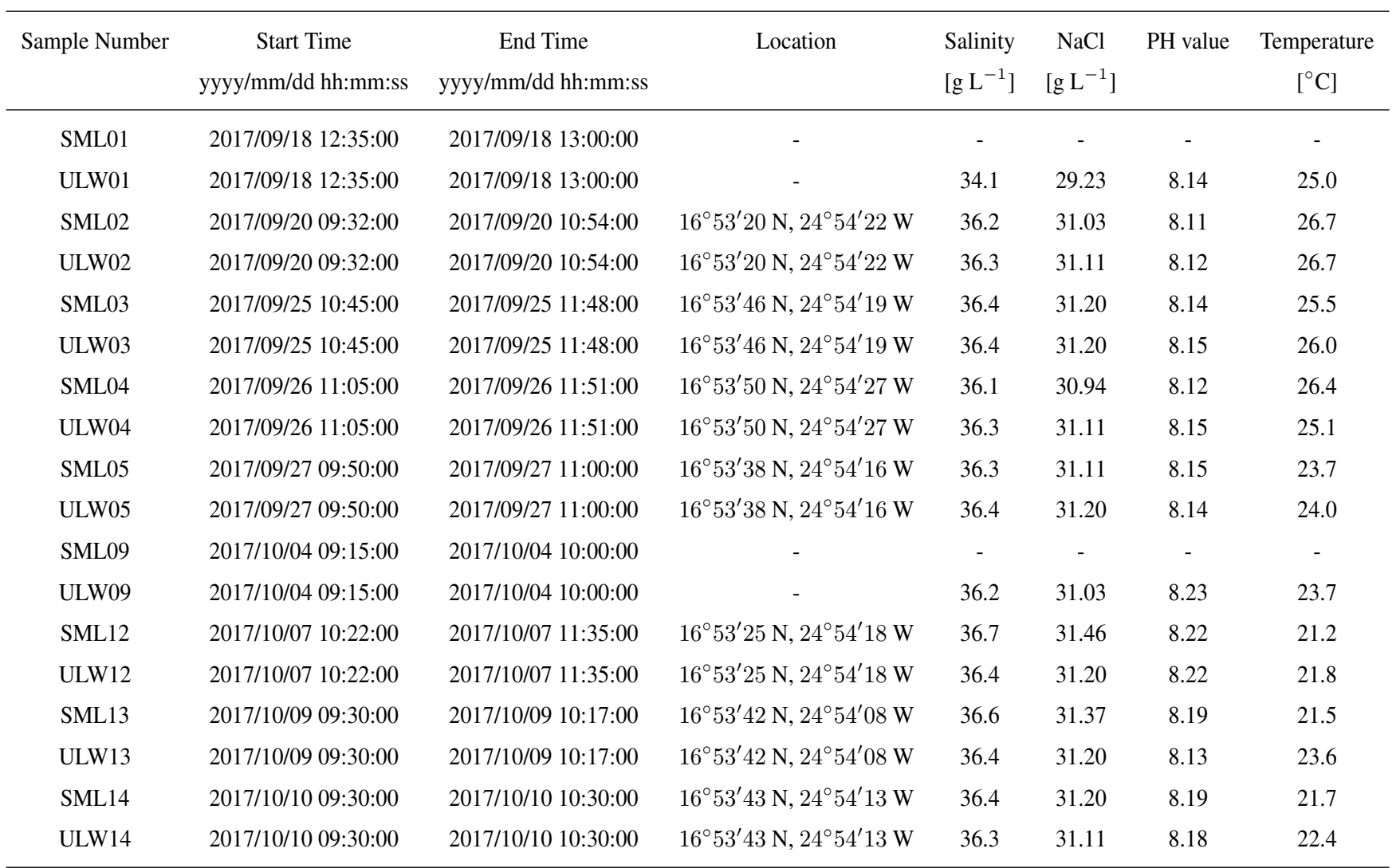




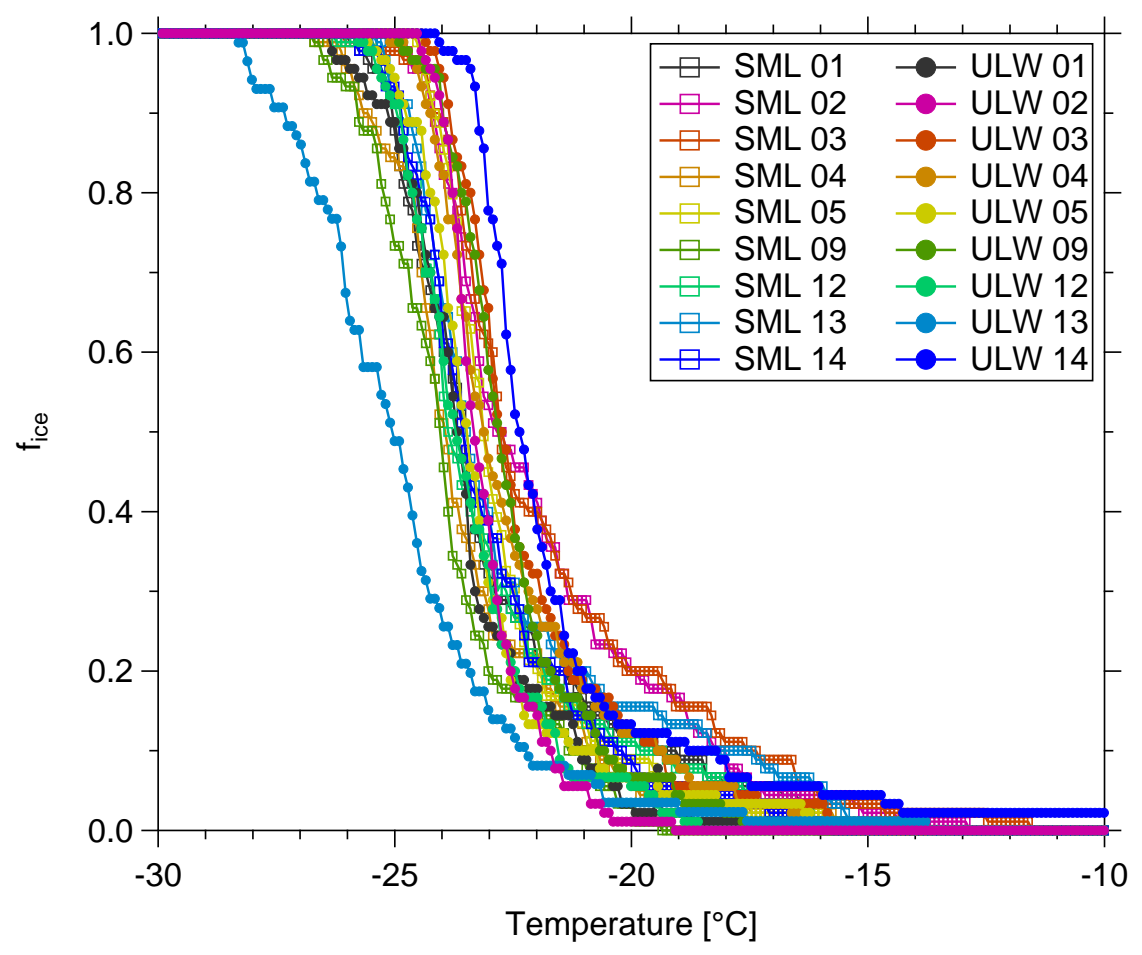

Figure S1. Frozen fraction ( $f_{\text {ice }}$ ) measured by LINA as a function of temperature in SML and ULW. All temperatures have been corrected for freezing point depression. 


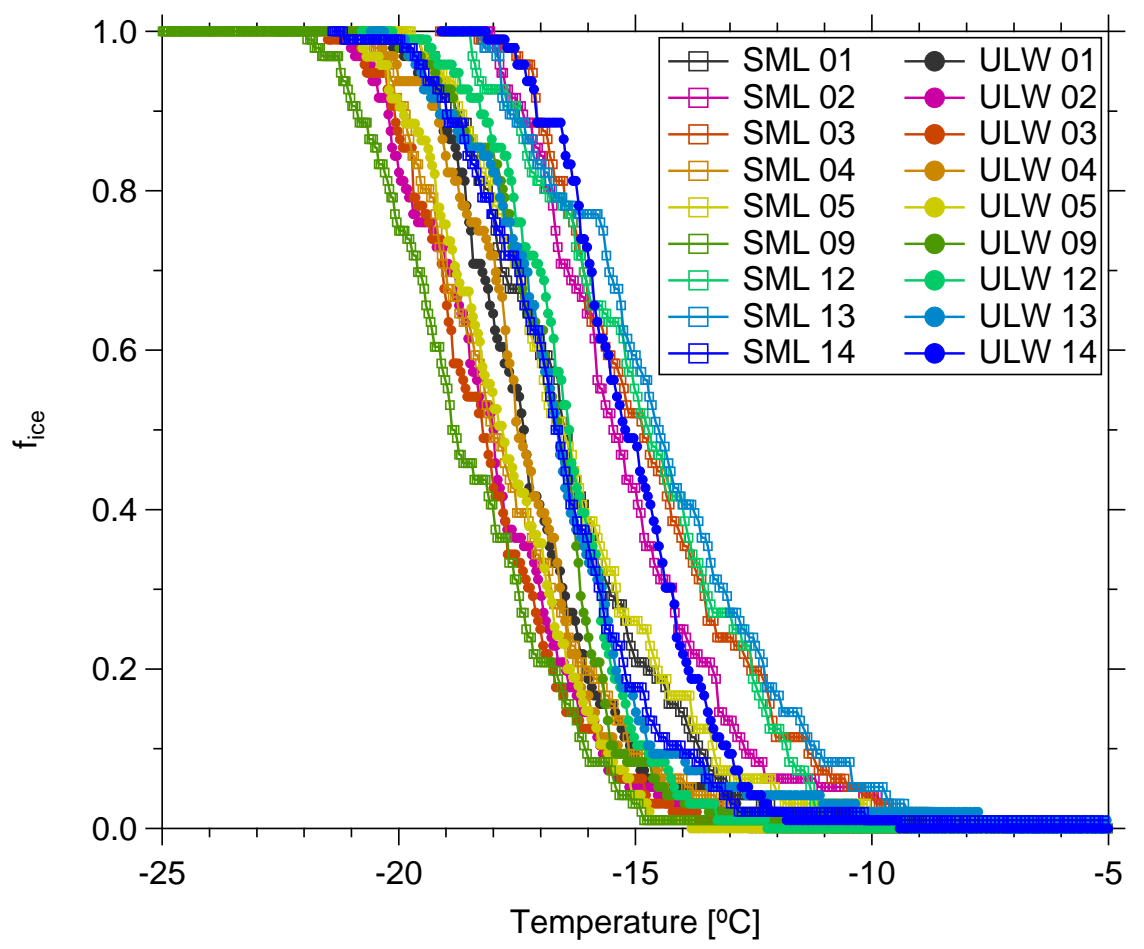

Figure S2. $f_{\text {ice }}$ measured by INDA as a function of temperature in SML and ULW. All temperatures have been corrected for freezing point depression. 


\section{S2 Filter samples}

\section{S2.1 CVAO PM 10}

Table S2. The information of $\mathrm{PM}_{10}$ filter samples at CVAO, including sample number, start time, end time, duration, total sampling volume, sampling volume per well, sodium $\left(\mathrm{Na}^{+}\right)$and chloride $\left(\mathrm{Cl}^{-}\right)$mass concentration, total particle surface area concentration $\left(A_{\text {total }}\right)$ and sample type.

\begin{tabular}{|c|c|c|c|c|c|c|c|c|c|}
\hline Sample Number & $\begin{array}{c}\text { Start Time } \\
\text { yyyy/mm/dd hh:mm:ss }\end{array}$ & $\begin{array}{c}\text { End Time } \\
\text { yyyy/mm/dd hh:mm:ss }\end{array}$ & $\begin{array}{c}\text { Duration } \\
\quad[\mathrm{h}]\end{array}$ & $\begin{array}{l}\text { Total Volume } \\
{\left[\operatorname{std~L}^{-1}\right]}\end{array}$ & $\begin{array}{c}\text { Volume Per Well } \\
\qquad\left[\operatorname{std~L}^{-1}\right]\end{array}$ & $\begin{array}{c}\mathrm{Na}^{+} \\
\mu \mathrm{g} \mathrm{m}^{-3}\end{array}$ & $\begin{array}{c}\mathrm{Cl}^{-} \\
\mu \mathrm{g} \mathrm{m}^{-3}\end{array}$ & $\begin{array}{c}A_{\text {total }} \\
\mu \mathrm{m}^{2} \mathrm{~cm}^{-3}\end{array}$ & Type \\
\hline CVAO1583 & 2017/09/19 21:00:00 & 2017/09/20 21:00:00 & 1439.34 & 660.289 & 33.6882 & 4.40 & 6.19 & 370 & $\mathrm{PM}_{10}$ \\
\hline CVAO1586 & 2017/09/23 16:00:00 & 2017/09/24 16:00:00 & 1439.34 & 660.289 & 33.6882 & 2.36 & 3.36 & 78 & $\mathrm{PM}_{10}$ \\
\hline CVAO1587 & 2017/09/24 16:00:00 & 2017/09/25 16:00:00 & 1439.34 & 660.289 & 33.6882 & 2.83 & 3.54 & 158 & $\mathrm{PM}_{10}$ \\
\hline CVAO1589 & 2017/09/26 16:00:00 & 2017/09/27 16:00:00 & 1439.61 & 661.462 & 33.7481 & 1.41 & 1.99 & 159 & $\mathrm{PM}_{10}$ \\
\hline CVAO1590 & 2017/09/27 16:00:00 & 2017/09/28 16:00:00 & 1439.71 & 661.644 & 33.7573 & 1.77 & 2.70 & 198 & $\mathrm{PM}_{10}$ \\
\hline CVAO1591 & 2017/09/28 16:00:00 & 2017/09/29 16:00:00 & 1439.73 & 661.420 & 33.7459 & 5.04 & 8.41 & 325 & $\mathrm{PM}_{10}$ \\
\hline CVAO1592 & 2017/09/29 16:00:00 & 2017/09/30 16:00:00 & 1439.73 & 660.289 & 33.6882 & 6.49 & 11.26 & 297 & $\mathrm{PM}_{10}$ \\
\hline CVAO1593 & 2017/09/30 16:00:00 & 2017/10/01 16:00:00 & 1439.73 & 660.821 & 33.7153 & 5.32 & 8.99 & 238 & $\mathrm{PM}_{10}$ \\
\hline CVAO1597 & 2017/10/03 16:00:00 & 2017/10/04 16:00:00 & 1439.71 & 660.629 & 33.7056 & - & - & 169 & $\mathrm{PM}_{10}$ \\
\hline CVAO1598 & 2017/10/05 16:00:00 & 2017/10/06 16:00:00 & 1439.55 & 659.264 & 33.6359 & 2.58 & 3.33 & 162 & $\mathrm{PM}_{10}$ \\
\hline CVAO1641 & 2017/10/06 16:00:00 & 2017/10/07 16:00:00 & 1439.73 & 658.670 & 33.6056 & 4.67 & 6.91 & 244 & $\mathrm{PM}_{10}$ \\
\hline CVAO1642 & 2017/10/07 16:00:00 & 2017/10/08 16:00:00 & 1439.71 & 661.187 & 33.7341 & 5.46 & 8.54 & 271 & $\mathrm{PM}_{10}$ \\
\hline CVAO1643 & 2017/10/08 16:00:00 & 2017/10/09 16:00:00 & 1439.71 & 659.785 & 33.6625 & 5.22 & 7.98 & 230 & $\mathrm{PM}_{10}$ \\
\hline CVAO1644 & 2017/10/07 17:00:00 & 2017/10/08 17:00:00 & & & & & & & Blind filter \\
\hline
\end{tabular}




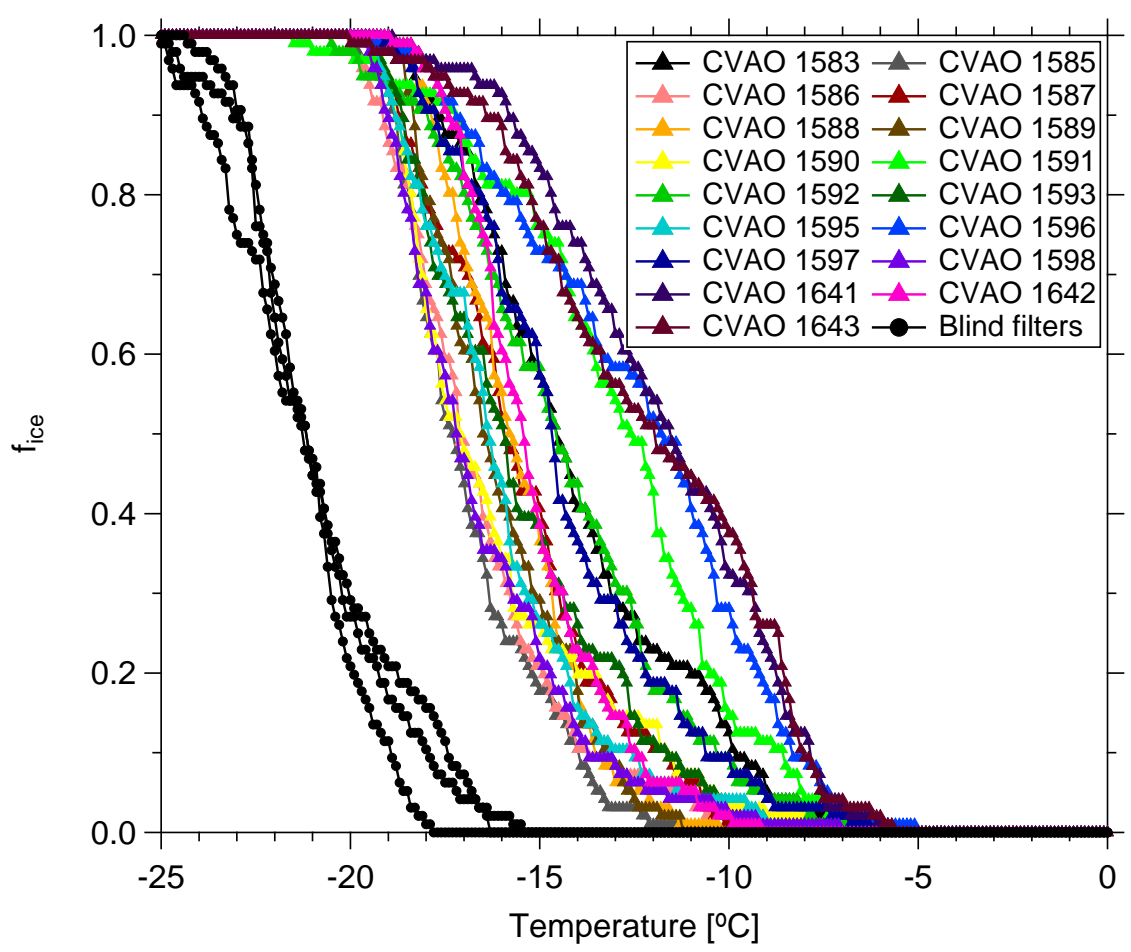

Figure S3. $f_{\text {ice }}$ measured by INDA (without background subtraction) as a function of temperature in CVAO PM 10 filters. $f_{i c e}$ of blind filters are shown by black dots. 


\section{S2.2 CVAO PM 1}

Table S3. The information of $\mathrm{PM}_{1}$ filter samples at CVAO, including sample number, start time, end time, duration, total sampling volume, sampling volume per well and sample type.

\begin{tabular}{|c|c|c|c|c|c|c|}
\hline Sample Number & $\begin{array}{c}\text { Start Time } \\
\text { yyyy/mm/dd hh:mm:ss }\end{array}$ & $\begin{array}{c}\text { End Time } \\
\text { yyyy/mm/dd hh:mm:ss }\end{array}$ & $\begin{array}{c}\text { Duration } \\
{[\mathrm{h}]}\end{array}$ & $\begin{array}{l}\text { Total Volume } \\
{\left[\operatorname{std~L}^{-1}\right]}\end{array}$ & $\begin{array}{l}\text { Volume Per Well } \\
\qquad\left[\operatorname{std} L^{-1}\right]\end{array}$ & Type \\
\hline CVAO924 & 2017/09/19 21:00:00 & 2017/09/20 21:00:00 & 1439.36 & 661.200 & 33.7347 & $\mathrm{PM}_{1}$ \\
\hline CVAO925 & 2017/09/21 21:00:00 & 2017/09/22 21:00:00 & 1439.36 & 661.200 & 33.7347 & $\mathrm{PM}_{1}$ \\
\hline CVAO926 & 2017/09/22 16:00:00 & 2017/09/23 16:00:00 & 1439.36 & 661.200 & 33.7347 & $\mathrm{PM}_{1}$ \\
\hline CVAO927 & 2017/09/23 16:00:00 & 2017/09/24 16:00:00 & 1439.36 & 661.200 & 33.7347 & $\mathrm{PM}_{1}$ \\
\hline CVAO928 & 2017/09/24 16:00:00 & 2017/09/25 16:00:00 & 1439.36 & 661.200 & 33.7347 & $\mathrm{PM}_{1}$ \\
\hline CVAO929 & 2017/09/25 16:00:00 & 2017/09/26 16:00:00 & 1439.21 & 664.115 & 33.8834 & $\mathrm{PM}_{1}$ \\
\hline CVAO930 & 2017/09/26 16:00:00 & 2017/09/27 16:00:00 & 1439.36 & 661.200 & 33.7347 & $\mathrm{PM}_{1}$ \\
\hline CVAO931 & 2017/09/27 16:00:00 & 2017/09/28 16:00:00 & 1439.36 & 661.200 & 33.7347 & $\mathrm{PM}_{1}$ \\
\hline CVAO932 & 2017/09/28 16:00:00 & 2017/09/29 16:00:00 & 1439.36 & 661.200 & 33.7347 & $\mathrm{PM}_{1}$ \\
\hline CVAO933 & 2017/09/29 16:00:00 & 2017/09/30 16:00:00 & 1439.36 & 661.200 & 33.7347 & $\mathrm{PM}_{1}$ \\
\hline CVAO934 & 2017/09/30 16:00:00 & 2017/10/01 16:00:00 & 1439.36 & 661.200 & 33.7347 & $\mathrm{PM}_{1}$ \\
\hline CVAO935 & 2017/09/29 16:00:00 & 2017/09/30 16:00:00 & & & & Blind filter \\
\hline CVAO936 & 2017/10/01 16:00:00 & 2017/10/02 16:00:00 & 1438.53 & 659.798 & 33.6632 & $\mathrm{PM}_{1}$ \\
\hline CVAO937 & 2017/10/02 16:00:00 & 2017/10/03 16:00:00 & 1439.55 & 660.255 & 33.6865 & $\mathrm{PM}_{1}$ \\
\hline CVAO938 & 2017/10/03 16:00:00 & 2017/10/04 16:00:00 & 1439.36 & 661.200 & 33.7347 & $\mathrm{PM}_{1}$ \\
\hline CVAO939 & 2017/10/04 16:00:00 & 2017/10/05 16:00:00 & 1439.36 & 661.200 & 33.7347 & $\mathrm{PM}_{1}$ \\
\hline CVAO940 & 2017/10/05 16:00:00 & 2017/10/06 16:00:00 & 1439.18 & 661.071 & 33.7281 & $\mathrm{PM}_{1}$ \\
\hline CVAO941 & 2017/10/06 16:00:00 & 2017/10/07 16:00:00 & 1439.58 & 662.336 & 33.7927 & $\mathrm{PM}_{1}$ \\
\hline CVAO942 & 2017/10/07 16:00:00 & 2017/10/08 16:00:00 & 1439.58 & 662.122 & 33.7817 & $\mathrm{PM}_{1}$ \\
\hline CVAO944 & 2017/10/08 16:00:00 & 2017/10/09 16:00:00 & 1439.55 & 660.377 & 33.6927 & $\mathrm{PM}_{1}$ \\
\hline
\end{tabular}




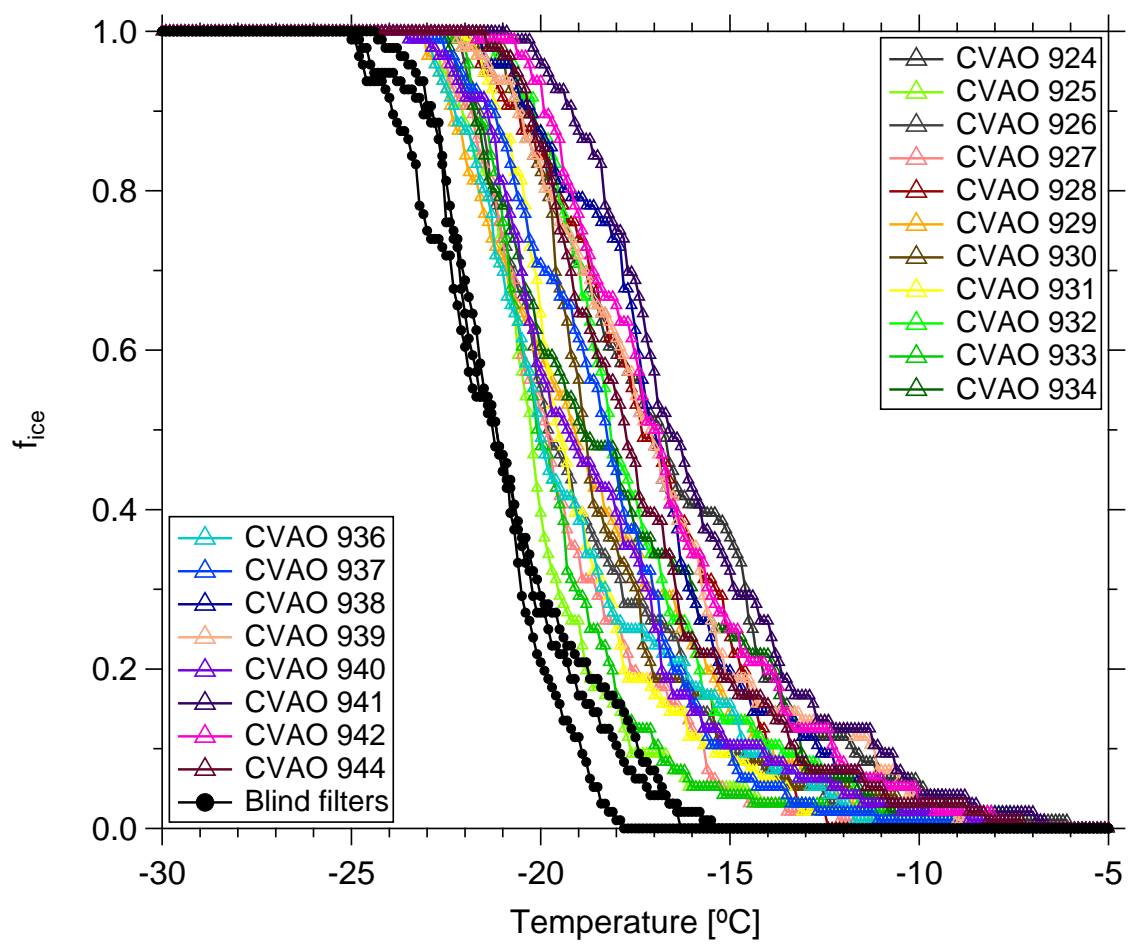

Figure S4. $\mathrm{f}_{\mathrm{ice}}$ measured by INDA (without background subtraction) as a function of temperature in CVAO PM $\mathrm{P}_{1}$ filters. $\mathrm{f}_{\text {ice }}$ of blind filters are shown by black dots. 


\section{S2.3 MV PM 10}

Table S4. The information of $\mathrm{PM}_{10}$ filter samples at MV, including sample number, start time, end time, duration, total sampling volume, sampling volume per well, cloud time (percent of the time MV was in cloud during the filter was sampled) and sample type.

\begin{tabular}{|c|c|c|c|c|c|c|c|}
\hline Sample Number & $\begin{array}{c}\text { Start Time } \\
\text { yyyy/mm/dd hh:mm:ss }\end{array}$ & $\begin{array}{c}\text { End Time } \\
\text { yyyy/mm/dd hh:mm:ss }\end{array}$ & $\begin{array}{c}\text { Duration } \\
{[\mathrm{h}]}\end{array}$ & $\begin{array}{l}\text { Total Volume } \\
{\left[\operatorname{std} \mathrm{L}^{-1}\right]}\end{array}$ & $\begin{array}{l}\text { Volume Per Well } \\
\qquad\left[\operatorname{std~L}^{-1}\right]\end{array}$ & $\begin{array}{c}\text { Cloud time } \\
{[\%]}\end{array}$ & Type \\
\hline MV1600 & 2017/09/21 16:39:00 & 2017/09/22 16:23:00 & 1382.86 & 601.870 & 30.7077 & $67.44 \%$ & $\mathrm{PM}_{10}$ \\
\hline MV1601 & 2017/09/22 16:23:00 & 2017/09/23 15:59:00 & 1418.31 & 615.998 & 31.4285 & $17.39 \%$ & $\mathrm{PM}_{10}$ \\
\hline MV1602 & 2017/09/23 15:59:00 & 2017/09/24 16:01:00 & 1440.60 & 625.035 & 31.8896 & $6.12 \%$ & $\mathrm{PM}_{10}$ \\
\hline MV1603 & 2017/09/24 16:01:00 & 2017/09/25 16:11:00 & 1449.61 & 629.660 & 32.1255 & $4.17 \%$ & $\mathrm{PM}_{10}$ \\
\hline MV1604 & 2017/09/25 16:13:00 & 2017/09/26 16:19:00 & 1444.90 & 627.655 & 32.0232 & $61.70 \%$ & $\mathrm{PM}_{10}$ \\
\hline MV1605 & 2017/09/26 16:20:00 & 2017/09/27 16:23:00 & 1440.58 & 627.381 & 32.0092 & $65.96 \%$ & $\mathrm{PM}_{10}$ \\
\hline MV1606 & 2017/09/27 16:23:00 & 2017/09/28 16:59:00 & 1464.99 & 637.541 & 32.5276 & $79.59 \%$ & $\mathrm{PM}_{10}$ \\
\hline MV1607 & 2017/09/28 17:01:00 & 2017/09/29 16:28:00 & 1406.21 & 611.922 & 31.2205 & $97.83 \%$ & $\mathrm{PM}_{10}$ \\
\hline MV1608 & 2017/09/29 16:30:00 & 2017/09/30 16:28:00 & 1676.36 & 760.265 & 38.7890 & $93.75 \%$ & $\mathrm{PM}_{10}$ \\
\hline MV1609 & 2017/10/01 19:02:00 & 2017/10/02 17:09:00 & 1326.63 & 576.405 & 29.4084 & $47.73 \%$ & $\mathrm{PM}_{10}$ \\
\hline MV1610 & 2017/10/02 17:09:00 & 2017/10/03 17:09:00 & 1439.36 & 624.715 & 31.8732 & $52.08 \%$ & $\mathrm{PM}_{10}$ \\
\hline MV1611 & 2017/10/03 17:10:00 & 2017/10/04 16:27:00 & 1396.11 & 606.390 & 30.9383 & $50.00 \%$ & $\mathrm{PM}_{10}$ \\
\hline MV1612 & 2017/10/04 16:27:00 & 2017/10/05 16:00:00 & 1408.61 & 613.421 & 31.2970 & $69.05 \%$ & $\mathrm{PM}_{10}$ \\
\hline MV1613 & 2017/10/05 16:00:00 & 2017/10/06 16:01:00 & 1441.46 & 627.486 & 32.0146 & $79.59 \%$ & $\mathrm{PM}_{10}$ \\
\hline MV1614 & 2017/10/06 16:03:00 & 2017/10/07 16:02:00 & 1439.46 & 625.832 & 31.9302 & $87.23 \%$ & $\mathrm{PM}_{10}$ \\
\hline MV1615 & 2017/10/07 16:02:00 & 2017/10/08 18:12:00 & 1439.36 & 627.485 & 32.0145 & $100.00 \%$ & $\mathrm{PM}_{10}$ \\
\hline MV1616 & 2017/10/08 18:13:00 & 2017/10/09 12:04:00 & 1071.60 & 467.526 & 23.8534 & $100.00 \%$ & $\mathrm{PM}_{10}$ \\
\hline
\end{tabular}




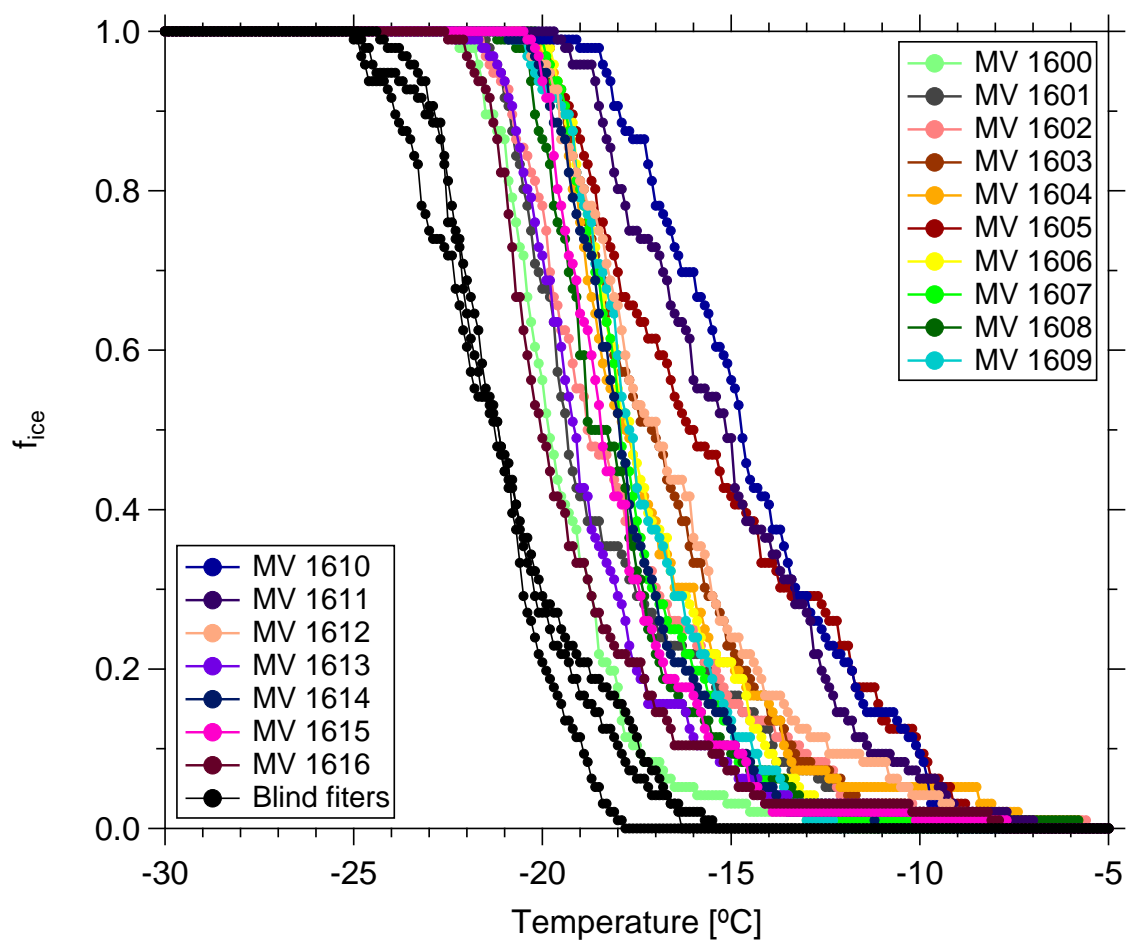

Figure S5. $f_{\text {ice }}$ measured by INDA (without background subtraction) as a function of temperature in MV PM 10 filters. $f_{i c e}$ of blind filters are shown by black dots. 


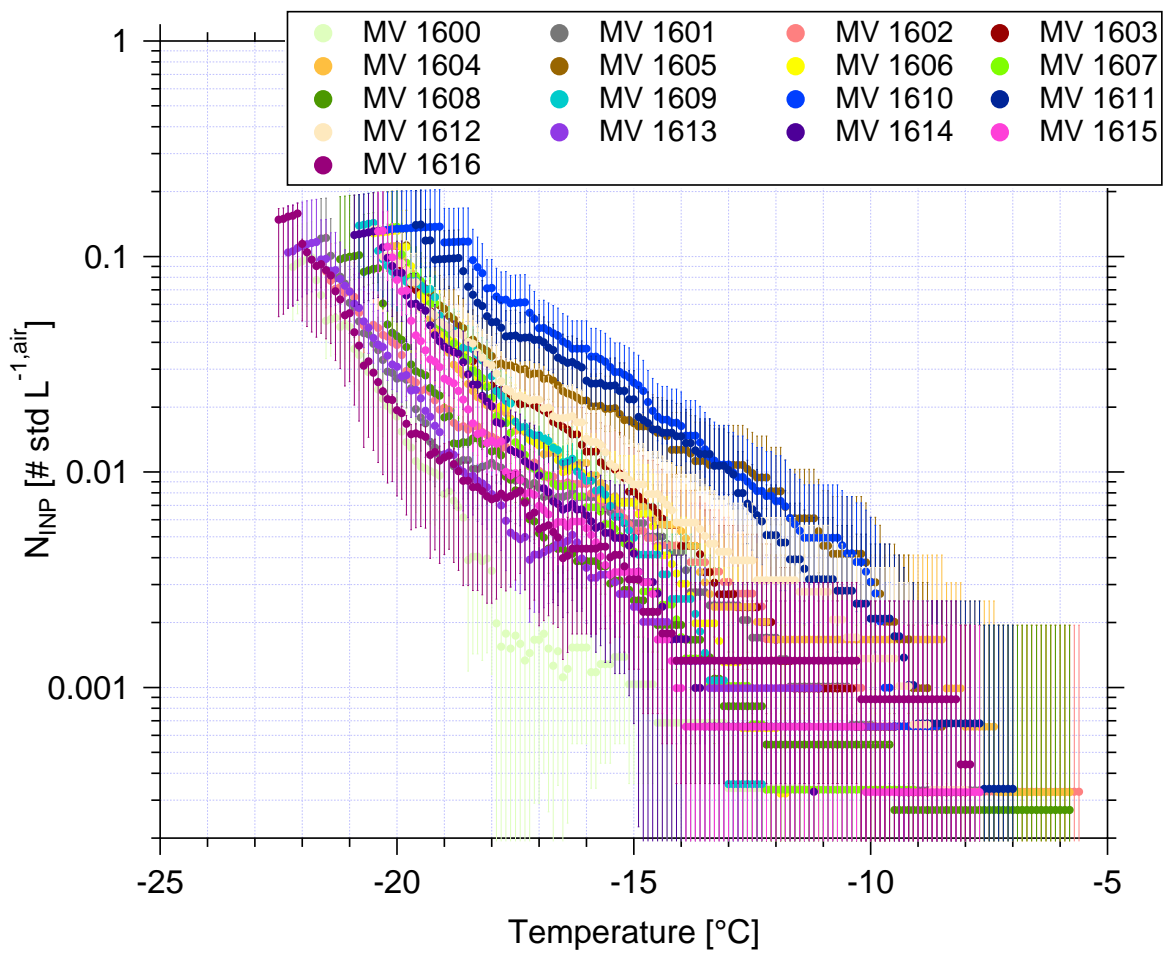

Figure S6. $N_{\text {INP }}$ as function of temperature in $\mathrm{MV} \mathrm{PM}_{10}$ filters. $N_{\mathrm{INP}}$ are background-corrected. Error bars show the $95 \%$ confidence interval. 


\section{S3 Cloud samples}

Table S5. The information of cloud water samples, including sample number, start time, end time, duration, volume, sodium (Na ${ }^{+}$) and chloride $\left(\mathrm{Cl}^{-}\right)$mass concentration and $N_{\mathrm{CCN}, 0.30 \%}$.

\begin{tabular}{|c|c|c|c|c|c|c|c|}
\hline Sample Number & $\begin{array}{c}\text { Start Time } \\
\text { yyyy/mm/dd hh:mm:ss }\end{array}$ & $\begin{array}{c}\text { End Time } \\
\text { yyyy/mm/dd hh:mm:ss }\end{array}$ & $\begin{array}{l}\text { Duration (h) } \\
\quad[\mathrm{h}]\end{array}$ & $\begin{array}{l}\text { Volume } \\
{[\mathrm{mL}]}\end{array}$ & $\begin{array}{c}\mathrm{Na}^{+} \\
\mathrm{mg} \mathrm{L}^{-1}\end{array}$ & $\begin{array}{c}\mathrm{Cl}^{-} \\
\mathrm{mg} \mathrm{L}^{-1}\end{array}$ & $\begin{array}{c}N_{\mathrm{CCN}, 0.30 \%} \\
\mathrm{~cm}^{-3}\end{array}$ \\
\hline Cloud01 & 2017/09/20 13:25:00 & 2017/09/20 18:20:00 & 4.92 & 185 & 8.44 & 15.51 & 551 \\
\hline Cloud03 & 2017/09/26 19:00:00 & 2017/09/27 08:00:00 & 13.00 & 435 & 8.32 & 14.15 & 387 \\
\hline Cloud04 & 2017/09/27 19:00:00 & 2017/09/28 07:30:00 & 12.50 & 544 & 5.00 & 9.27 & 239 \\
\hline Cloud05 & 2017/09/28 19:00:00 & 2017/09/29 07:30:00 & 12.50 & 537 & 14.18 & 24.57 & 560 \\
\hline Cloud11 & 2017/10/04 19:00:00 & 2017/10/05 07:30:00 & 12.50 & 150 & 46.11 & 70.30 & 481 \\
\hline Cloud12 & 2017/10/05 07:45:00 & 2017/10/05 17:38:00 & 9.88 & 78 & 22.75 & 36.99 & 494 \\
\hline Cloud13 & 2017/10/05 17:40:00 & 2017/10/05 20:10:00 & 2.50 & 133 & 16.97 & 25.23 & 442 \\
\hline Cloud14 & 2017/10/05 20:10:00 & 2017/10/05 23:30:00 & 3.33 & 131 & 17.31 & 24.36 & 473 \\
\hline Cloud15 & 2017/10/05 23:30:00 & 2017/10/06 04:00:00 & 4.50 & 120 & 21.85 & 31.95 & 491 \\
\hline Cloud16 & 2017/10/06 04:05:00 & 2017/10/06 08:00:00 & 3.92 & 120 & 16.87 & 19.77 & 445 \\
\hline Cloud19 & 2017/10/06 18:00:00 & 2017/10/07 06:30:00 & 12.50 & 537 & 18.34 & 29.10 & 482 \\
\hline Cloud20 & 2017/10/07 06:48:00 & 2017/10/07 10:48:00 & 4.00 & 88 & 28.19 & 41.54 & 510 \\
\hline Cloud24 & 2017/10/08 19:00:00 & 2017/10/09 07:00:00 & 12.00 & 537 & 24.54 & 32.46 & 625 \\
\hline
\end{tabular}

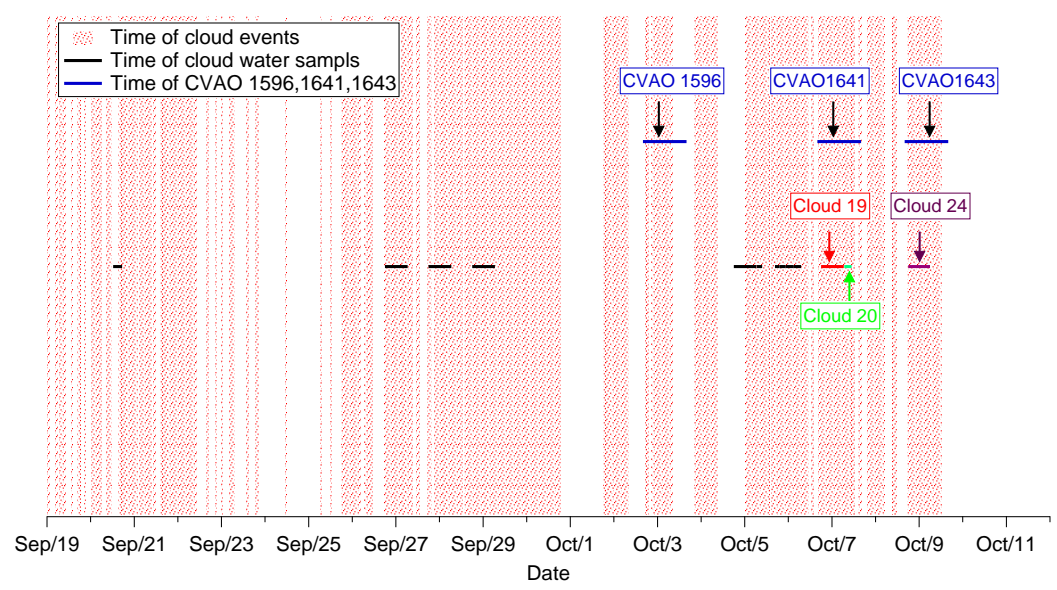

Figure S7. Times during which MV was in clouds (in red shadows) and the sampling time of all cloud water and that of some selected CVAO $\mathrm{PM}_{10}$ filters. 


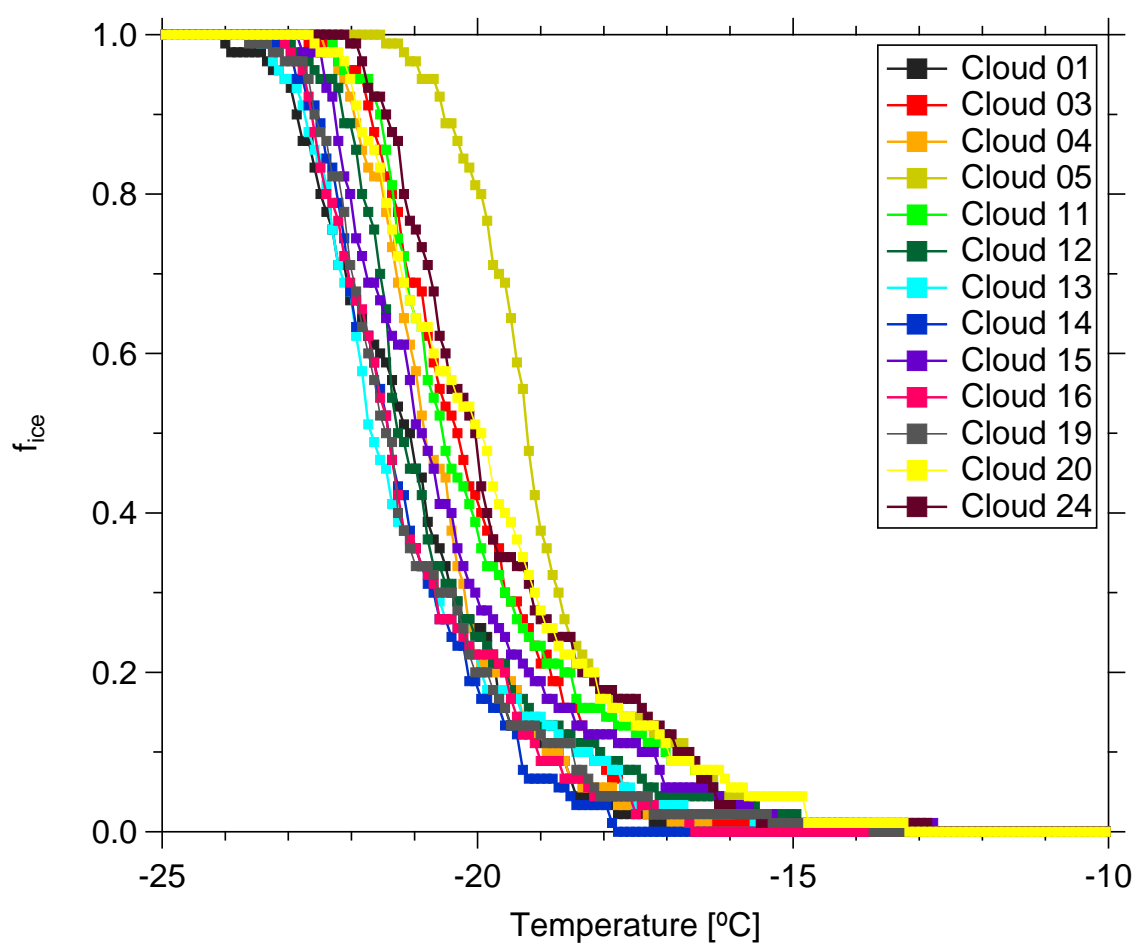

Figure S8. $f_{i c e}$ measured by LINA as a function of temperature in cloud water.

\section{S4 Particle surface area size distribution}

A thorough aerosol characterization has been done during the measurement campaign, and is described in detail in Gong et al. (2019). Fig. S10 shows the median particle surface area size distribution (PASD) for the whole campaign. Error bars show the 75th and 25 th percentiles. Two different modes were observed, i.e., a small mode $(30-500 \mathrm{~nm})$ and a larger mode $(500 \mathrm{~nm}-10$ $\mu \mathrm{m})$. The larger mode particle surface area is about 3 times higher than the small mode. Based on the PASD, the concentrations for the total surface area of the particles were calculated. The total particle surface area concentration $\left(A_{\text {total }}\right)$ varied from 35 to $824 \mu \mathrm{m}^{2} \mathrm{~cm}^{-3}$, with a median of $116 \mu \mathrm{m}^{2} \mathrm{~cm}^{-3}$. The averaged $A_{\text {total }}$ during each CVAO PM 10 sampling period varied from 78 to $370 \mu \mathrm{m}^{2} \mathrm{~cm}^{-3}$ (summarized in Tab. S2). Based on airborne measurements in the Saharan dust layer, Price et al. (2018) found $A_{\text {total }}$ mainly above 100 with a maximum of $688 \mu \mathrm{m}^{2} \mathrm{~cm}^{-3}$, which is higher than values found for this study, likely due to the fact that Cape Verde is at some distance to the Sahara and also that less strong dust events were sampled. 


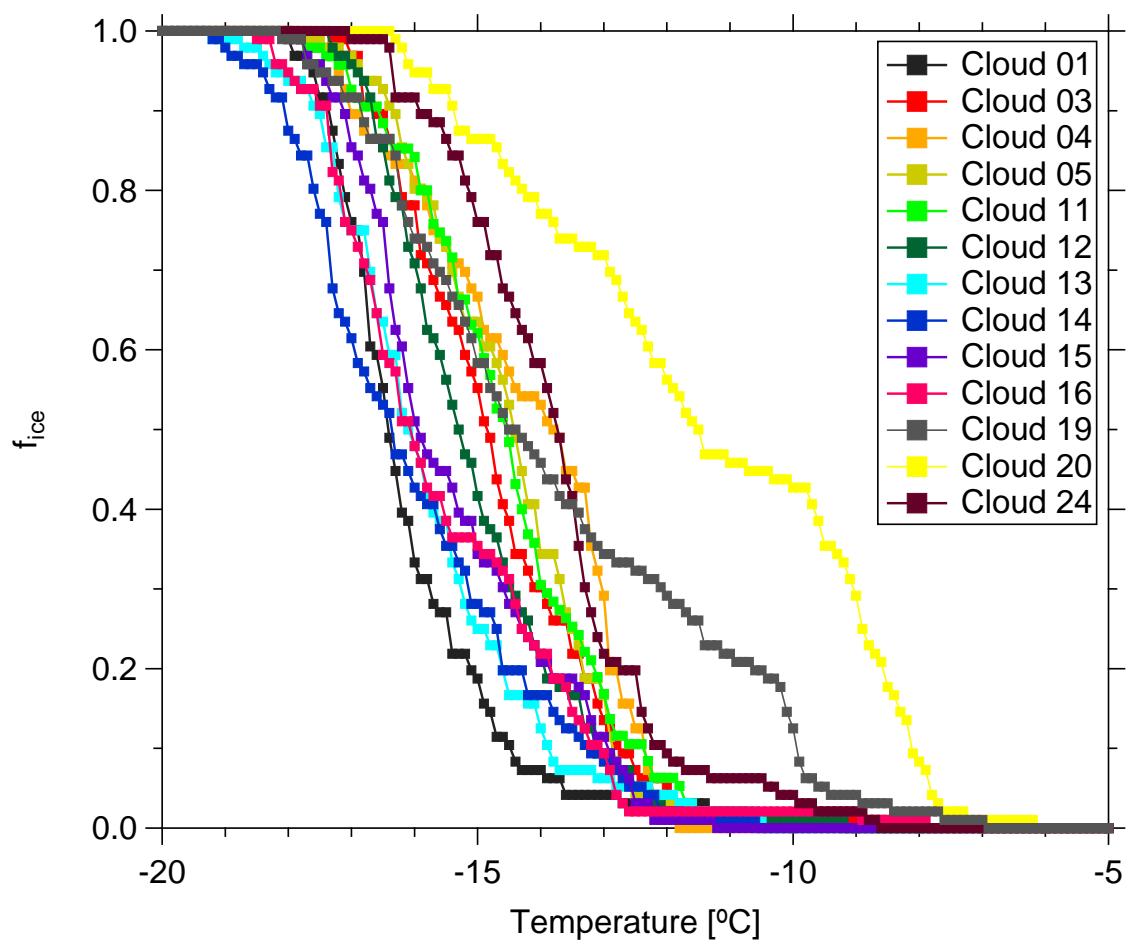

Figure S9. $f_{\text {ice }}$ measured by INDA as a function of temperature in cloud water.

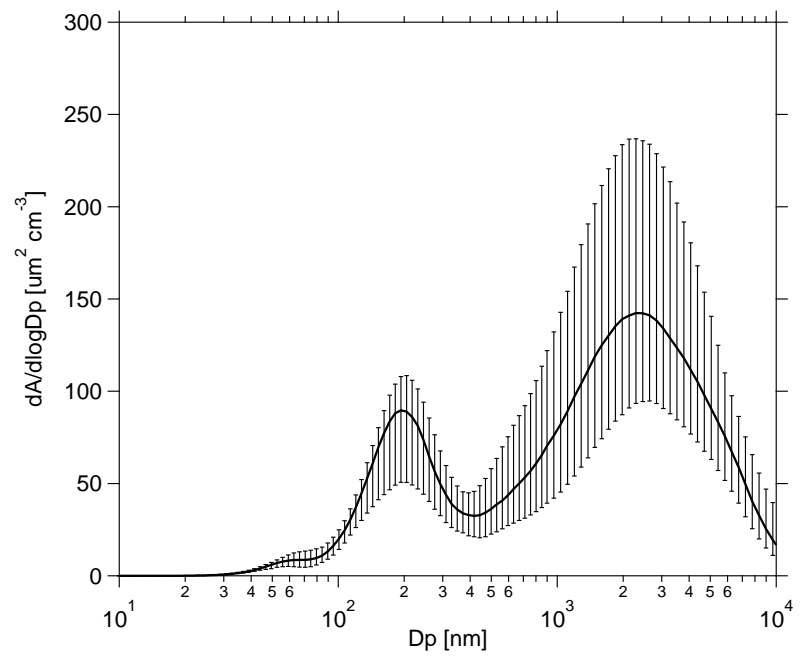

Figure S10. The median PASD during the whole campaign. The error bar indicates the range between the 75 th and 25 th percentiles. 


\section{References}

Gong, X., Wex, H., Voigtländer, J., Fomba, K. W., Weinhold, K., van Pinxteren, M., Henning, S., Müller, T., Herrmann, H., and Stratmann, F.: Characterization of aerosol particles at Cape Verde close to sea and cloud level heights - Part 1: particle number size distribution, cloud condensation nuclei and their origins, Atmos. Chem. Phys. Discuss., 2019, 1-31, https://doi.org/10.5194/acp-2019-585, https:// www.atmos-chem-phys-discuss.net/acp-2019-585/, 2019.

Price, H. C., Baustian, K. J., McQuaid, J. B., Blyth, A., Bower, K. N., Choularton, T., Cotton, R. J., Cui, Z., Field, P. R., Gallagher, M., Hawker, R., Merrington, A., Miltenberger, A., Neely III, R. R., Parker, S. T., Rosenberg, P. D., Taylor, J. W., Trembath, J., VergaraTemprado, J., Whale, T. F., Wilson, T. W., Young, G., and Murray, B. J.: Atmospheric Ice-Nucleating Particles in the Dusty Tropical Atlantic, Journal of Geophysical Research: Atmospheres, 123, 2175-2193, https://doi.org/doi:10.1002/2017JD027560, https://agupubs. onlinelibrary.wiley.com/doi/abs/10.1002/2017JD027560, 2018. 